\title{
A Product-Service System Approach to Telehealth Application Design
}

\author{
Paul Flores-Vaquero, Ashutosh Tiwari, Jeffrey Alcock, Windo Hutabarat and Chris Turner* \\ Manufacturing and Materials Department, Cranfield University, Bedfordshire, UK \\ Corresponding Author: \\ Chris Turner, Manufacturing and Materials Department, Cranfield University, Bedfordshire, \\ UK \\ Email: c.j.turner@cranfield.ac.uk
}

\begin{abstract}
A considerable proportion of current point-of-care devices do not offer a wide enough set of capabilities if they are to function in any telehealth system. There is a need for intermediate devices that lie between healthcare devices and service networks. The development of an application is suggested that allows for a smartphone to take the role of an intermediate device. This research seeks to identify the telehealth service requirements for long term condition management using a Product-Service System (PSS) approach. The use of PSS has proven to be a suitable methodology for the design and development of telehealth smartphone applications.
\end{abstract}

\section{Keywords}

Product-Service System (PSS), Smartphone Application, Intermediate Devices.

\section{Introduction}

The management of long term health conditions or chronic diseases is an ongoing concern for public healthcare organisations in Europe. Within the UK over 15 million people in England are suffering from at least one long term condition, defined as "a health problem that can't be cured but can be controlled by medication or other therapies". ${ }^{1}$ The management of long-term conditions creates high economic and human cost for health institutions, especially when it entails avoidable hospitalisations.

The deployment of telehealth systems that enable people with long-term conditions to manage their health at home could be the means towards cost-effective high quality care services. These kinds of telehealth systems rely on sensor-equipped healthcare devices (point-of-care devices) that keep track of patient's conditions and transmit this information to a monitoring service.

At present there is a general lack of informatics capabilities in existing healthcare devices, such devices do not record data in an exchangeable format and are difficult to interface with intermediary systems; this limits their use in telehealth applications. There is a need for 
Intermediate Devices (IDs) that address this informatics gap. IDs lie between healthcare devices and the service network allowing the interaction between patients and health professionals. ${ }^{2}$ Both healthcare devices and IDs are usually proprietary, which leads to interoperability issues, so an open source platform would be preferable to provide telehealth services. ${ }^{2}$ From this perspective, a recent increase in smartphone usage leads to an opportunity where smartphone applications can be designed and built that transform smartphones and tablets into telehealth IDs. Implementing Product-Service System (PSS) in telehealth opens a new opportunity to change the traditional healthcare delivery model. The PSS is a business model that is defined as "a marketable set of products and services capable of jointly fulfilling a user's need. ${ }^{3}$ The product/service ratio in this set can vary, either in terms of function fulfilment or economic value". ${ }^{4}$ It is suggested that the PSS concept can help obtain informatics service requirements for such applications. This paper explores the possibility of applying a PSS approach to the design of a telehealth ID smartphone application.

The need for telehealth services has been acknowledged by governmental institutions. The European Commission has found essential to apply information and communication technologies in healthcare sector across the European Union to provide more efficient healthcare services for European citizens ${ }^{5}$.

In the UK the Technology Strategy Board has also announced a $f 23$ million investment for the Dallas - Delivering Assisted Living Lifestyles at Scale- programme. This programme aims to show how assisted living can promote well-being, and provide independent living technologies and services. ${ }^{6}$ The devices used in this programme all output data in a form that can be read by the Dallas portal applications. ${ }^{7}$ In comparison with such telehealth systems the application developed for this research is potentially capable of being used with a wide range of both new and existing hardware devices as it acts as an intermediary device. This is especially important in healthcare systems where a wide range of devices may be in use and compatibility may be an issue. Such a smartphone device is flexible in terms of the user interface it can display to a user and type of data it can capture.

Even where medical devices are wireless capable it is still possible that a role for an intermediary device could still exist. In the work of Furniss et al. ${ }^{8}$ where the use of wireless glucometers in the UK National Health Service was examined, from an ergonomic standpoint, it was noted that a number of medical staff were unsure of what data was being uploaded and there were additional factors identified with the difficulty in the use of such devices by the patient at home. In such cases an intermediary device could display the data being uploaded and the success or failure of the transmission. In addition it could act as a real time guide to the use of the glucometer by users unfamiliar with its operation. It was also the case that the glucometers came with a note taking function, for the text based recording of patient details and observations, that was generally not used. It was put by Funrniss et al. ${ }^{8}$ that the note taking function should be redesigned and allow for two way communication with medical staff for example. Such text based functionality is readily available via a smartphone intermediary device with a suitable software application; with the benefit that many more people are familiar with the use of such phones. 


\section{A PSS Approach}

The healthcare sector has always been conceived as an area where PSSs have a huge potential. However, this sector has had limited research interest. One such research work is presented by Hareva et al. ${ }^{9}$ who provide a smartphone based iridology application to predict the health of a patent through an examination of their iris. The application is entirely phone based and can at present be used to predict four disease types.

In telehealth PSS terms the Point of Care (POC) device would be the product. The level of servitization of such a product would depend on the medicine informatics capabilities that the device offers. This may determine whether the device could be part of a PSS without any redesign, or whether there is a need to add any kind of product capability. ${ }^{10}$ The level of servitization would also determine at which level (product, use or result oriented) the PSS would operate.

Some services that could be provided by POC devices taking a PSS approach are: ${ }^{11}$

- Test management: Reminders for the patients to carry out tests.

- Medication management: Reminders for the patients to take their medication.

- Lifestyle management: An option for patients to enter their diet and lifestyle habits.

- Feedback (condition monitoring): A report on patient results and trends along with advice of professionals in order to improve patients' health conditions.

- Alarm management: Alarm triggering when there is any unexpected event (extreme result), prompting the user to contact a health professional or contacting health professionals automatically.

These functions are the service requirements for any result-oriented telehealth PSS.

\section{Adopted PSS Methodology}

One of the major decisions when developing a PSS is to initiate a suitable PSS design methodology. In this context, where a telehealth PSS has to be designed, the Use cases \& Scenarios methodology was followed. ${ }^{12}$ This methodology permits identifying the interaction between the elements involved in the PSS, and suggests methods to model use cases and scenarios. Finally, it presents the way to develop a service blueprint from which service requirements for the PSS can be obtained.

The design process of a PSS should cover the following phases: ${ }^{12}$

1. Actor networks identification

2. PSS envisioning: Use cases and scenarios.

3. PSS structure representation.

\section{Actor Networks Identification}

The design of integrated products and services involves both social and technological aspects. This means that the design of the PSS should be based on the combination of several factors such as people, technological artefacts and cultural frames. 
Both direct and indirect participants in the development of the PSS are relevant social groups. ${ }^{13} \mathrm{~A}$ framework of criteria to analyse each of the individuals from these social groups has been proposed by Bijker. ${ }^{14}$ In this research, these criteria were used to generate a set of user profiles within the telehealth system. These profiles were created according to users' key problems and requirements (Table 1 ). 
Table 1 Telehealth PSS main actors' profiles

\begin{tabular}{|c|c|c|c|}
\hline Actor & Characteristics & Key problems & Requirements \\
\hline \multirow[t]{4}{*}{ Patient } & People suffering from one & Unnecessary visits to the & Personal health monitoring \\
\hline & or more long term condition & doctor, lack of an & system \\
\hline & that need regular tracking & information system to store & \\
\hline & of their conditions & data & \\
\hline \multirow[t]{4}{*}{ Carer } & People in charge of the & Responsible for many & Patient monitoring system \\
\hline & well-being of patients when & patients, excessive amount & \\
\hline & patients need caring & of patients' data & \\
\hline & services & & \\
\hline Health & Professionals that provide & Lack of an information & Information system to track \\
\hline \multirow[t]{3}{*}{ Professional } & healthcare services in a & system to keep track of & patients' data \\
\hline & systematic way to a number & patients' data & \\
\hline & of patients & & \\
\hline \multirow[t]{6}{*}{ Health Institution } & Public or private institution & Increasing cost of & Technological \\
\hline & that provides health related & healthcare delivery due to & infrastructure to cost- \\
\hline & services & aging population, & effectively manage \\
\hline & & unnecessary & patients' health \\
\hline & & hospitalisations, duplicated & \\
\hline & & tests, etc. & \\
\hline
\end{tabular}


From these profiles, it is possible to understand the possible interaction between each group by analysing their key problems and requirements.

\section{PSS envisioning: use cases and scenarios}

Analysing possible PSS scenarios, understanding case studies and defining requirements for a PSS are essential tasks that need to be accomplished in this stage. In this phase, a simplified description of the flow of events, conditions and actors involved in the telehealth service system use case was created (Table 2). From this description, it was possible to visualise the information flow for the telehealth service to be designed. 
Table 2 Simplified description of a use case for the telehealth service

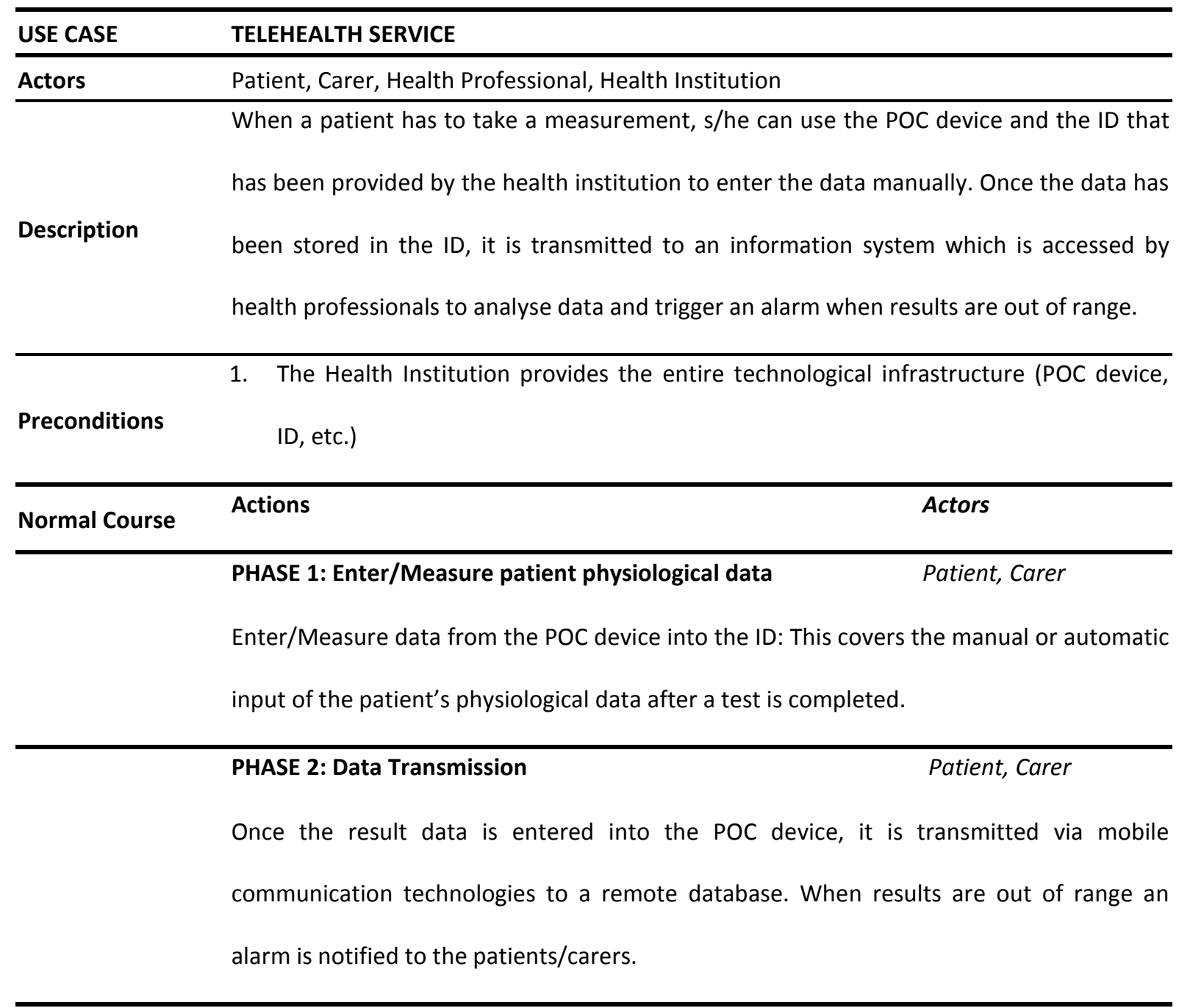

\section{PHASE 3: Data Analysis}

Health Professional

After the data is stored, the results in the database can be accessed through a web portal. This web portal is accessible by both patients and health professionals. Whenever a test result is out of range an alarm is notified to the health professionals.

PHASE 4 : Feedback

Patient, Carer, Health

\section{Professional}

The health professional can give feedback to the patients/carers periodically, or just after an alert has been sent via e-mail, SMS, phone call or having an in person appointment depending on the situation. 
Use case identification task can be supported by scenario modelling tools, such as IDEF0, to gain better understanding of the system, especially of the flow of events and information exchange process. Based on the representation method drawn from the work of Griscenko, ${ }^{11}$ it was possible to represent the telehealth PSS use case for the smartphone application. Fig 1 represents such a use case developed for managing diabetes. In Fig 1 patients can access the portal to have real-time access to view results and how they have been analysed and view online information and other tools. In turn the portal sends alerts and reminders to the patient. 


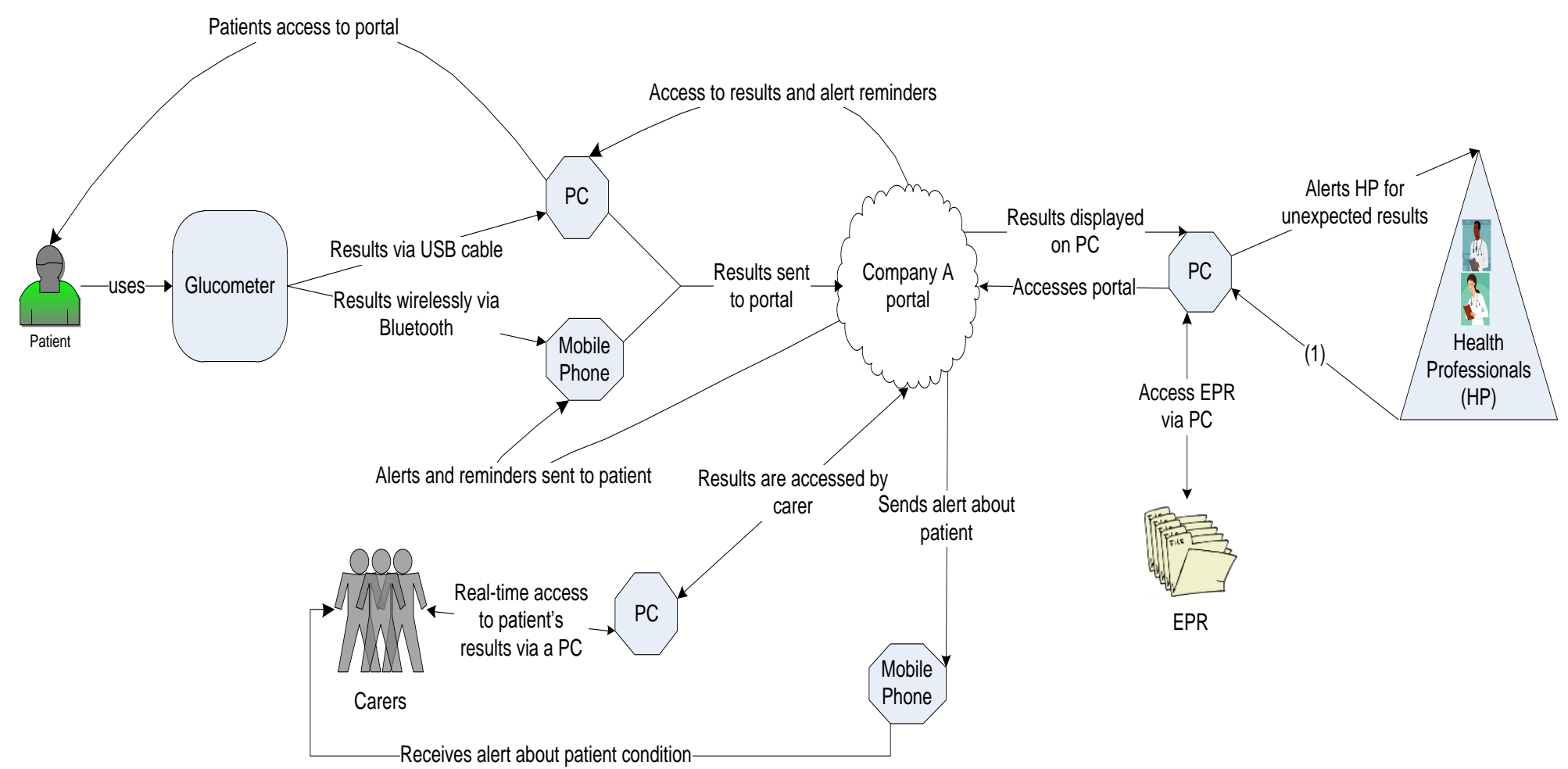

(1)

Real-time access to patient's results

Analyses patient's results

Can see an overview of the patient results

Can alter patient's medication

Fig 1 Telehealth system for diabetes management ${ }^{11}$ 
The main entity in this model is the ID that in this scenario would be the smartphone application. It is also necessary to notice that in this model there are two main users for the ID; patients and carers. As it is explained in the use case description, carers will only take part in the system when patients' health conditions require it.

\section{PSS structure representation}

Having a graphical representation standard becomes essential as PSS has to be represented in a way that is understandable for everyone. ${ }^{10}$ However, graphic notations alone are not enough to represent all the elements of a PSS. A methodology called "service blueprinting" has been proposed. ${ }^{15,16}$ In a typical service blueprint there are five components: ${ }^{17}$

- Customer actions: Includes the steps that customers go through as part of service delivery.

- Onstage contact: Includes all the interactions that occur face-to-face between the customer and the ones delivering the service.

- Backstage contact: Includes every action that occurs and is not visible for the customer.

- Support processes: Includes the activities carried out by people outside the system but that are necessary in order for the service to be delivered.

- Physical evidence: Includes the physical elements that customers come in contact with for each customer action.

Taking these elements into account, the service blueprint for the smartphone application was developed (shown in Appendix A). Analysing this service blueprint along with the telehealth scenario model of the previous section permitted the identification of the main service requirements for the telehealth smartphone application. This service blueprint was developed with reference to the work of Bordas-Eddy. ${ }^{18}$

\section{Application description}

The application works on the basis of users that suffer from long term conditions taking results from POC devices and storing these results on a remote database through a smartphone application. The test result can be entered into the application manually or transmitted directly from the POC device wirelessly using Bluetooth technology. Then, the result is transmitted and stored into a remote database via $3 \mathrm{G}$ mobile communication.

One of the potential issues of this application is the impossibility to transmit a result because communication channels are not available. For this reason, it is possible to check if the result has been stored properly in the database by accessing the web portal which displays the data stored in the database through the application.

The application gives the opportunity to manage the most common long term conditions. Hence, it is possible to use the application with a large number of POC devices, permitting patients who suffer from more than one chronic disease to have a multi disease management system in only one application. 


\section{Telehealth Smartphone Application Development}

The following design considerations for telehealth smartphone application development are put forward by the authors of this paper:

- Open architecture: Excessive dependence on proprietary healthcare devices and communication protocols limits the potential benefits of telehealth.

- Communications usage: Telehealth applications work on the basis of triggering emergency alerts when dangerous situations arise. Thus, it is essential that these functionalities do not depend on the availability of specific limited communication services. The smartphone must also be kept in range of the POC device for continuity of data transmission.

- Multi disease support: Telehealth smartphone application market shows a lack of applications offering multi disease support.

- Patient adaptation: Knowledge about long term conditions changes constantly.

- Responsiveness and feedback: Telehealth applications work on the basis of activating alarms when exceptional situations arise.

- Supportive healthcare information: Supplying the geo-location of health related points of interest (e.g. the location of the nearest pharmacies).

- Platform adaptation: The application must be implemented for a wide range of operating systems.

The operating system that was chosen to develop the smartphone application is Android. Android is a Linux-based operating system, which was especially created for mobile phones at the beginning, but which has been extended to tablets, MP3 players, netbooks or televisions. It is an open source operating system, with Application Programming Interfaces (APIs) libraries written in C and applications running on Java. ${ }^{19}$

The (UML) Universal Modelling Language (UML is a modelling language designed for the meta-representation of object oriented software providing a comprehensive notation set) a representation of the developed telehealth smartphone application is available in Appendix B.

\section{Case Study: Blood Pressure Monitoring Service}

A case study was undertaken in order to validate the approach outlined in this paper. An application based on the design considerations (outlined in the previous section) was developed in this case study. The case study presents a complete service for a blood pressure monitoring telehealth system. The POC device used in the case study is the UA767 PBT blood pressure monitor manufactured by A\&D Company Limited. ${ }^{20}$ An interview with the manufacturers of this device, Saludnova, was conducted as part of the validation of the methodology developed in this research. The blood pressure monitoring device was selected as it was the one that complied with the widest range of national and international health regulations (the device also carries the CE (Conformité Européenne) mark of conformance with European Union standards). The UA-767PBT blood pressure monitor is equipped with Bluetooth wireless communication technology (Fig 2). 


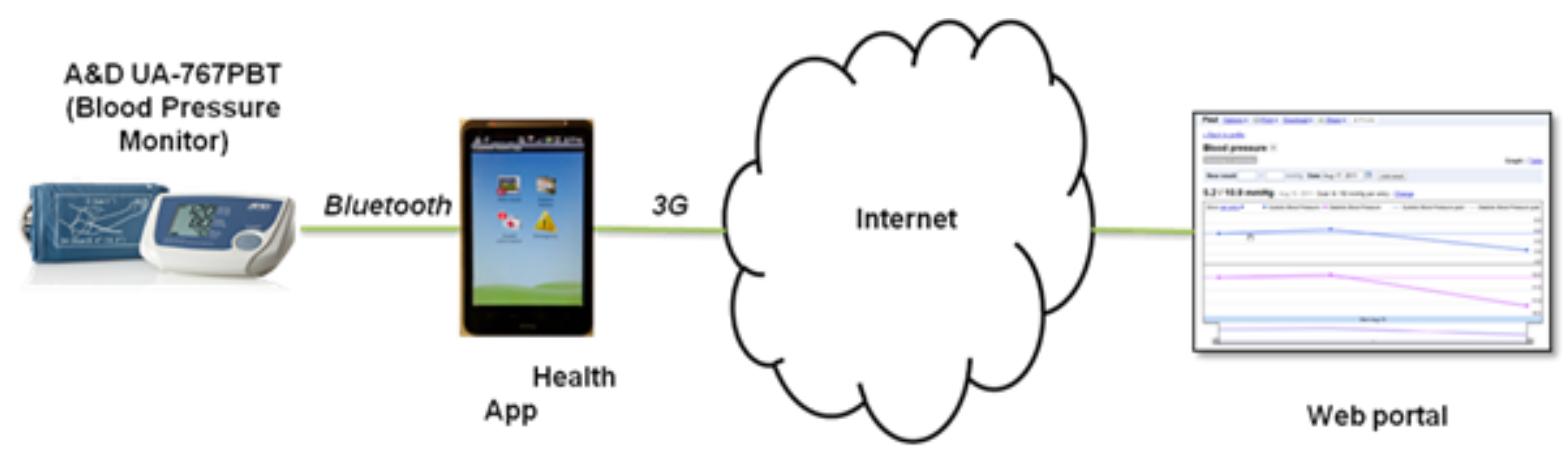

Fig 2 Case study schema

The main User Interface (UI) screen (a screen provided for a user to enter data and operate the computer application) (Fig 3) is developed using self-explanatory icons to make the application as user friendly as possible. Having a self-explanatory UI gains special importance considering that a significant proportion of potential users, especially older people, have never used smartphones.

The icons in the main screen represent the principal services offered by the application.

- New result: Allows users to store a new result from the POC device, either manually or via Bluetooth communication technology.

- Patient History: Allows users to access their patient history to visualise a list of all the results stored in the system.

- Health Information: Provides users with health related information to locate health related points of interest via GPS capability.

- Emergency: Allows users to make an emergency call or send an automatic emergency SMS with the coordinates of the user.

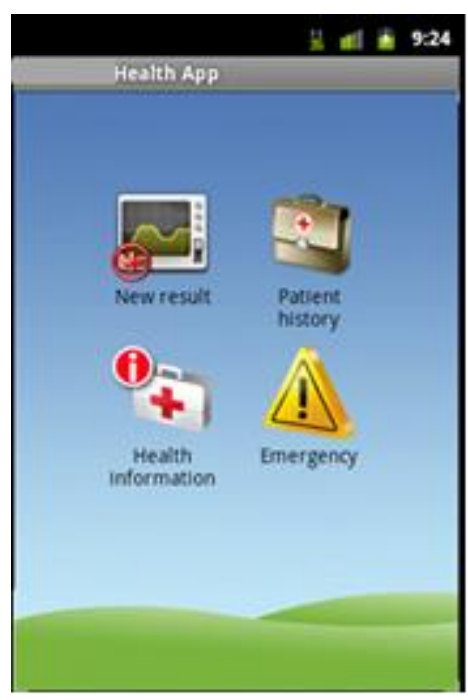

Fig 3 Telehealth application main screen 
By pressing the New Result icon it is possible to enter and store a new test result in the system. The result can be entered manually in the system or automatically through the Bluetooth capability of the blood pressure monitor (Fig 4).

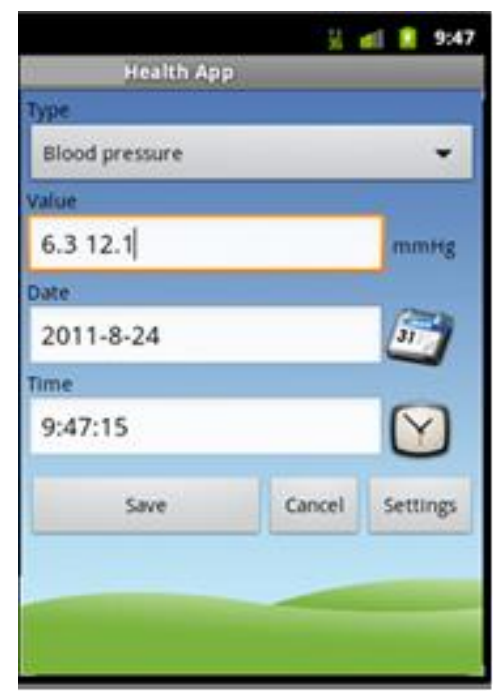

Fig 4 Receiving and storing a new result

Once the test result is obtained, the result could be sent to a platform such as Microsoft HealthVault ${ }^{21}$ by pressing the save button. In this case study, the application has its own predefined risk levels for both systolic and diastolic pressure $(14 / 9 \mathrm{mmHg})$. Whenever a test result is over these pre-established limits, a message is sent to a pre-set emergency contact person. The test result data that has already been stored can immediately be displayed in a health portal such as that provided by Microsoft. In particular it can be linked to the charting services for blood pressure and can access data and records stored within the health portal relating to medical history. Health portals can also be used by professionals to access patient's data, have real time information on results or analyse patterns in the results.

\section{Conclusions}

The blood pressure monitoring service is only one sample of the multiple long term conditions that can be managed by this application. The case study presented in this paper is just one example of the important benefits that such systems can offer to Healthcare Institutions. While smartphones are starting to be used in telehealth scenarios, ${ }^{22}$ the use of PSS to develop a telehealth smartphone application is a novel contribution to the area of telehealth application design. The Telehealth PSS design methodology and literature review supported the identification of the state-of-the-art in the design of telehealth smartphone applications. In this process, multiple methods and techniques, such as actor network identification, use case and scenarios modelling and service blueprinting, have been utilised. In this research, the PSS concept has allowed for design considerations and service requirements to be incorporated into telehealth smartphone applications from the start. A number of areas for future research have also been identified by the authors:

- Customisation of medical knowledge for a more patient oriented telehealth service. 
- Providing the application with decision making intelligence so that multiple parameters can be taken into account, in cases where patients suffer from more than one condition.

- Analysis on how this telehealth monitoring system would be adapted to fit with an enterprise healthcare system.

The application development process has derived significant benefits from the use of a PSS design and development methodology. Without set benchmarks, standards, or methods for the design of telehealth smartphone applications, the PSS concept has proven to be a suitable methodology. With an emerging concept as telehealth PSS, it is likely that research to achieve the full potential of this approach will require further investment from Health Institutions.

\section{References}

1. Department of Health. Improving quality of life for people with long term conditions, https://www.gov.uk/government/policies/improving-quality-of-life-for-people-withlong-term-conditions (2012, accessed 19th September 2013).

2. Adeogun $\mathrm{O}$, Tiwari $\mathrm{A}$, and Alcock JR. Capabilities of proprietary intermediate telehealth devices. Telemedicine and e-Health 2011; 17: pp 712-721.

3. Bains TS, Lightfoot HW, Evans S, Neely A, Greenrough R, Peppard J, Roy R, Shehab E, Braganza A, Tiwari A, Alcock JR, Angus JP, Bastl M, Cousens A, Irving P, Johnson M, Kingston J, Lockett H, Martinez V, Michele P, Tranfield D, Walton IM, Wilson H. Stateof-the-art in product-service systems. In Proceedings of ImechE, , Part B: J. Engineering Manufacture 2007; 221: pp 1543-1552.

4. Goedkoop MJ, van Halen CJG, te Riele HRM, Rommens, PJM. Product service systems, ecological and economic basis. PricewaterhouseCoopers N.V. / Pi!MC, Storrm C.S., Pre consultants, 1999

5. European Commission Digital Agenda for Europe (2014), Putting patients in the driving seat: A digital future for healthcare, available at http://ec.europa.eu/digitalagenda/en/news/putting-patients-driving-seat-digital-future-healthcare (accessed 09 September 2014).

6. DALLAS - Delivering Assisted Living Lifestyles at Scale, http://www.telecare.org.uk/industry/dallas (accessed 09 September 2014)

7. Technology Strategy Board (2011), DALLAS - Delivering Assisted Living Lifestyles at Scale, available at: http://www.innovateuk.org/content/competition/dallasdelivering-assisted-living-lifestyles-at-sc.ashx (accessed 09 September 2014)

8. Furniss, D., Masci, P., Curzon, P., Mayer, A., \& Blandford, A. 7 Themes for guiding situated ergonomic assessments of medical devices: A case study of an inpatient glucometer. Applied ergonomics 2014, 45(6), 1668-1677.

9. Hareva, D. H., Lukas, S. and Suharta, N. O. The smart device for healthcare service: Iris diagnosis application, 2013 Eleventh International Conference on ICT and Knowledge Engineering, Amsterdam, Netherlands. 
10. Ajai O, Tiwari A and Alcock JR. Evaluation of the state-of-the-art in informatics in glucometers, Informatics for Health and Social Care 2009; 34: pp 171-179.

11. Griscenko, N. (2012). Comparison and evaluation of the Telehealth systems using a discrete event simulation, MSc Thesis, Cranfield University.

12. Morelli N. Developing new product service systems (PSS): Methodologies and operational tools, Journal of Cleaner Production 2006; 14: pp 1495-1501.

13. Bijker WE. Of bicycles, bakelites, and bulbs: Toward a theory of sociotechnical change, Inside technology, MIT Press, Cambridge, MA, 1997

14. Bijker WE, Hughes TP and Pinch TJ. The social construction of technological systems: new directions in the sociology and history of technology, MIT Press, Cambridge, MA, 1987

15. Shostack GL. How to design a service, European Journal of Marketing 1982; 16: pp 49-63.

16. Shostack GL. Designing services that deliver, Harvard Business Review 1984; 62: p 133.

17. Bitner M. Ostrom AL. and Morgan FN. Service blueprinting: A practical technique for service innovation, California Management Review, 2008; 50: p 66.

18. Bordas-Eddy A. Providing an integrated supportive service system design framework for new digital innovations, Masters Thesis, Cranfield University, Cranfield, UK, 2011.

19. Android developers (2014), Android, available at: http://developer.android.com/about/index.html (accessed 09 September 2014).

20. A\&D Company Limited . UA-767PBT, http://www.aandd.jp/products/medical/bluetooth/ua_767pbt.html (accessed 09 September 2014).

21. Microsoft. Microsoft Health Vault, https://www.healthvault.com/gb/en (accessed 09 September 2014).

22. Lee HR. Yoo SK. Jung SM. Kwon NY. and Hong CS. A Web-based mobile asthma management system, Journal of Telemedicine and Telecare 2005; 11: pp 56-59. 


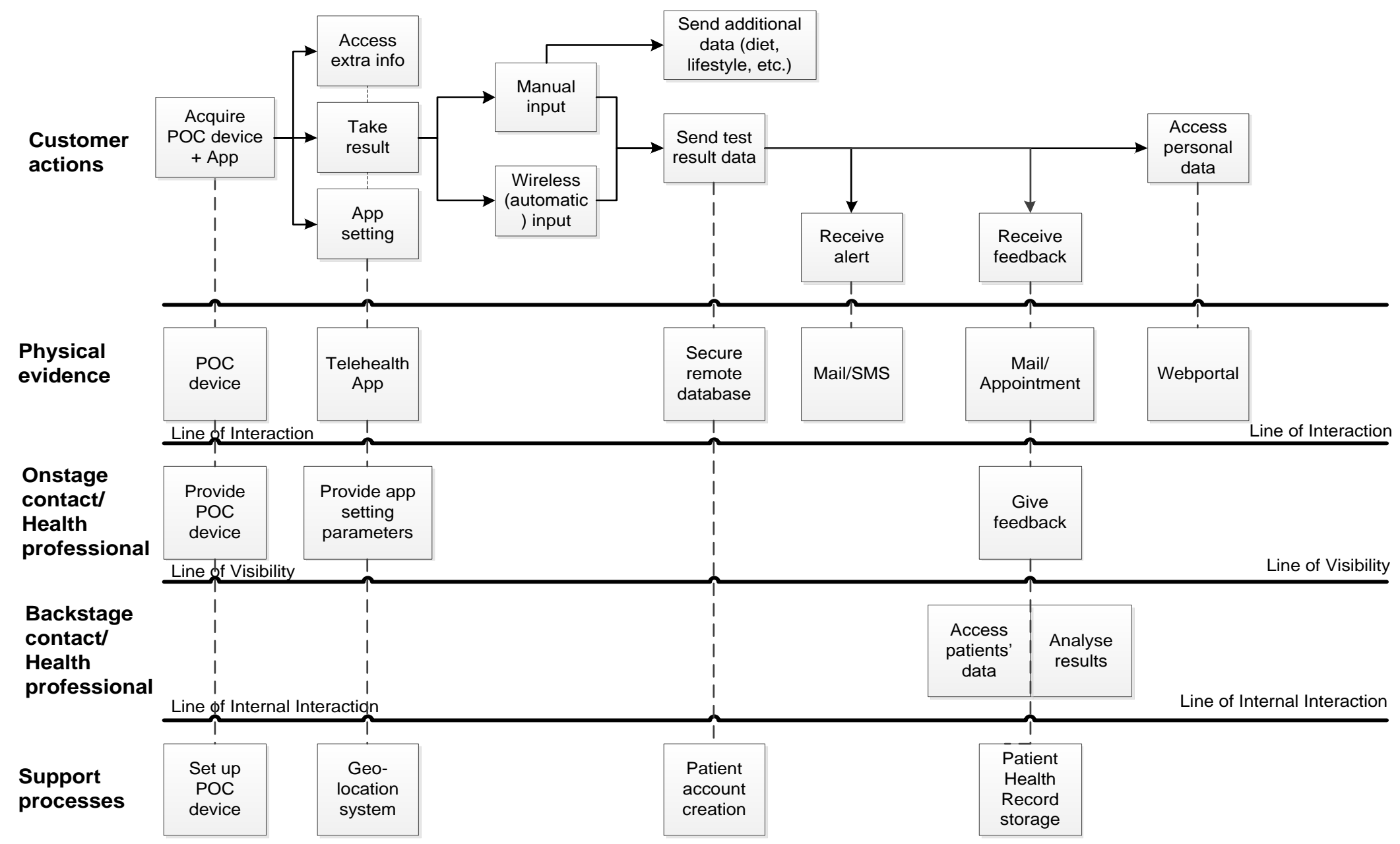

Telehealth PSS service blueprint 
Appendix B

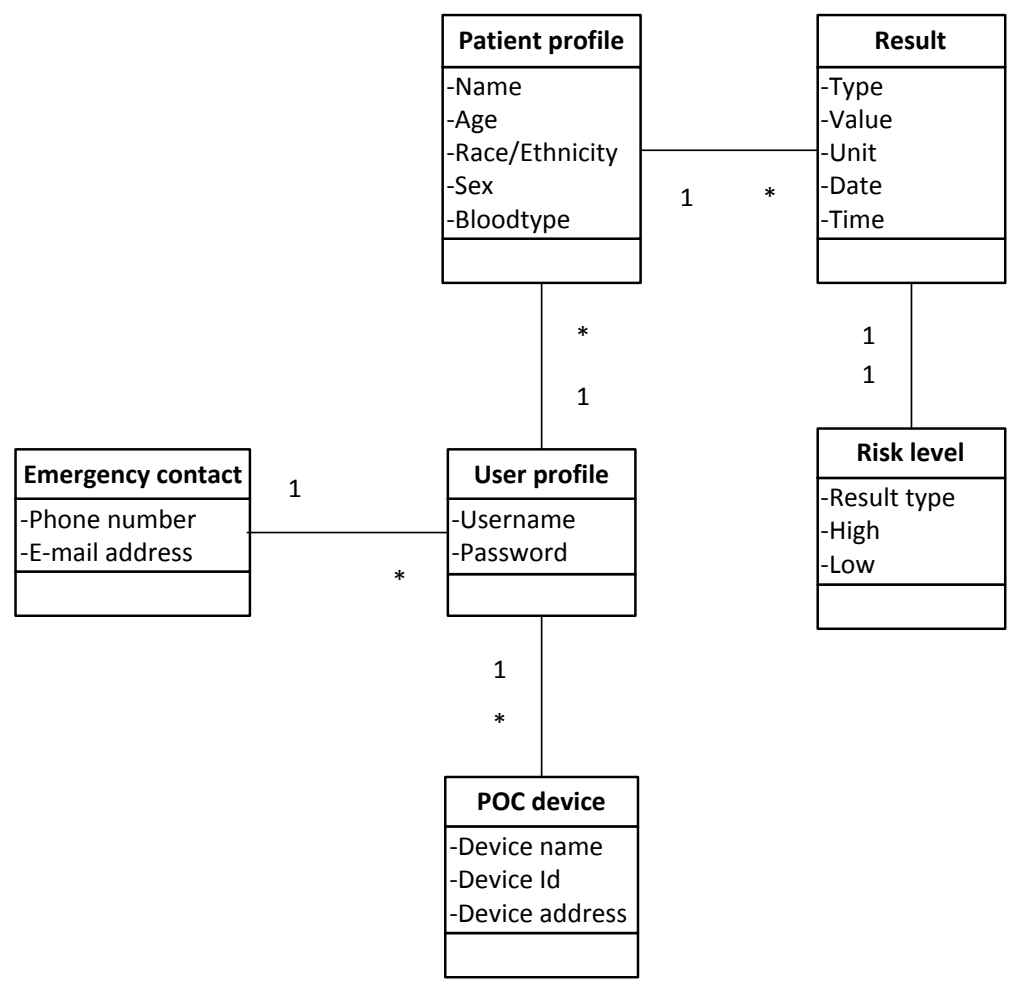

Smartphone application UML representation 
2016-06-30

\section{A product-service system approach to telehealth application design}

Flores-Vaquero, Paul

SAGE

Flores-Vaquero et. al (2016) A product-service system approach to telehealth application design, Health Informatics Journal, Vol. 22, Iss. 2, pp. 321-332

http://dx.doi.org/10.1177/1460458214558313

Downloaded from Cranfield Library Services E-Repository 